African Crop Science Journal by African Crop Science Society is licensed under a Creative Commons Attribution 3.0 Uganda License. Based on a work at www.ajol.info/ and www.bioline.org.br/cs DOI: https://dx.doi.org/10.4314/acsj.v27i3.5

\title{
INHERITANCE OF RESISTANCE TRAITS TO AFRICAN STEM BORER IN GRAIN SORGHUM
}

\author{
P.W. MUTURI, P. RUBAIHAYO ${ }^{1}$ and M. MGONJA ${ }^{2}$
}

University of Embu, Department of Agricultural Resource Management, P. O. Box 6 - 60100, Embu, Kenya

${ }^{1}$ Makerere University, Department of Agricultural Production, P. O. Box 7062, Kampala, Uganda ${ }^{2}$ Alliance for a Green Revolution in Africa, P. O. Box 34441, Dar es Salaam, Tanzania Corresponding author: muturi.phyllis@embuni.ac.ke

(Received 25 April 2018; accepted 9 August 2019)

\begin{abstract}
The African stem borer (Busseola fusca [Fuller]) is an important insect pest of cereals, mainly maize, sorghum and millets. The pest causes up to $80 \%$ reduction in grain yield, depending on the pest population in the field, cultivar and the management practices employed. This reduction in yield translates into food insecurity, especially in semi-arid lands (ASALs) where sorghum is cultivated by small holder farmers. This study investigated the inheritance of resistance traits to the African stem borers in grain sorghum in Kenya. The experimental material consisted of seventeen sorghum lines with varying levels of resistance to the African stem borer. The mating design employed was North Calorina Design 2, where 15 sorghum lines were used as females on two males. The crosses were evaluated in two seasons at University of Embu farm in 2011/2012 short and long seasons replicated twice. Artificial infestation with African stem borer neonates was done 30 days after planting using a camel brush. Data collected included stem borer damage and agro-morphological traits. Genetic analyses were performed using a line $\mathrm{x}$ tester method using Genstat statistical software. It was found that both additive and non-additive gene effects were important in conditioning resistance traits to the African stem borer. This implies that expression of high leaf glossiness, plant vigour and bloom waxiness in F1 hybrids is conditioned by additive genes and these traits can be used as morphological markers to select for resistance to the borer in sorghum. ICSB 464 x ICSB 473 was the best combiner for stem borer resistance and grain yield. Parents ICSA 464, ICSB 464 and ICSB 474, were among the good combiners for resistance to the stem borer. These parents can be utilised in developing superior sorghum hybrids resistant to the insect pest.
\end{abstract}

Key Words: Gene action, heritability, Sorghum bicolor

\section{RÉSUMÉ}

Le foreur africain de tiges (Busseola fusca [Fuller]) est un insecte ravageur important pour les céréales, principalement le maïs, le sorgho et le mil. L' insecte réduit jusqu'à $80 \%$ le rendement en grains, en 
fonction de la population d' insecte dans le champ, du cultivar et des pratiques de gestion employées. Cette réduction de rendement cause une insécurité alimentaire, en particulier dans les zones semiarides (ASAL) où le sorgho est cultivé par de petits agriculteurs. Cette étude a examiné la transmission des caractères de résistance aux foreurs africain de tiges chez le sorgho à grains au Kenya. Le matériel expérimental consistait en dix-sept lignées de sorgho présentant différents niveaux de résistance au foreur africain de tiges. Le plan d'accouplement utilisé était le North Calorina Design 2, où 15 lignées de sorgho étaient utilisées en tant que femelles sur deux mâles. Les croisements ont été évalués au cours de deux saisons à la ferme de l'Université d'Embu en 2011/2012 des saisons courtes et longues ont été répliquées deux fois. L'infestation artificielle de nouveau-nés avec des tétranyques a été réalisée 30 jours après la plantation à l'aide d'une brosse à chameaux. Les données collectées comprenaient les dommages causés par les foreurs de tiges et les caractéristiques agro-morphologiques. Les analyses génétiques ont été effectuées à l'aide d'une méthode de test de ligne x utilisant le logiciel statistique Genstat. Il a été constaté que les effets génétiques additifs et non additifs étaient importants pour conditionner les caractères de résistance au foreur de tiges. Cela implique que l'expression de gènes additifs conditionne l'expression d'une brillance élevée des feuilles, de la vigueur des plantes et du fart de la floraison chez les hybrides F1. Ces caractères peuvent être utilisés comme marqueurs morphologiques pour sélectionner la résistance au foreur du sorgho. ICSB 464 x ICSB 473 était le meilleur combineur pour la résistance des foreurs de tiges et le rendement en grains. Les parents ICSA 464, ICSB 464 et ICSB 474 figuraient parmi les bons combinateurs pour la résistance au foreur. Ces parents peuvent être utilisés pour développer des hybrides de sorgho supérieurs résistants à l'insecte ravageur.

Mots Clés: Action des gènes, héritabilité, Sorghum bicolor

\section{INTRODUCTION}

Sorghum (Sorghum bicolor (L.) Moench) is an important indigenous nutritious cereal crop, cultivated by small holder farmers in Subsaharan Africa (Sharma et al., 2006). Production of sorghum is constrained by biotic, abiotic and social-cultural challenges. The African stem borer Busseola fusca Fuller (Lepidopteran: Noctuidae) is recognised as an economically important insect pest of sorghum, maize and millet (Kfir et al., 2002). Busseola fusca is associated with significant grain yield losses ranging between $15-80 \%$, depending on the pest population, time of infestation, variety and agroecosystem (Wale et al., 2007). The quantitative loses translate into food insecurity at the house hold levels, especially during periods where maize fails due to prolonged drought. Qualitative losses include low marketability of the grain, thus the produce does not fetch premium prices in the market. Busseola fusca inflicts damage through leaf feeding, dead heart, exit holes and stem tunneling (Chabi-Olaye et al., 2005). The $B$. fusca larvae remain inside the stems, and thus, are protected from insecticides and natural enemies (Muhammad et al., 2009).

In Kenya, Busseola fusca is endemic in high altitudes and co-exits in mid-altitude zones with Chilo partellus Swinhoe, (Lepidopteran: Crambidae), which is an introduced pest from Asia (Kfir et al., 2002). In Kenya, much research has been accorded to $C$. partellus, consequently neglecting indigenous economically important stem borers such as the African stem borer. Research on management of $B$. fusca in sorghum has mainly focused on biological control and cultural control, predominantly intercropping, residue management, fertiliser use, and recently, genetic engineering (Chabi et al., 2005; Markus and Gurling, 2006; Amsalu et al., 2008).

Host plant resistance is an efficient approach in managing insect pests, especially in Africa where subsistence farmers practice little or no pest management in cereals (Sharma 
et al., 2006). Combining host plant resistance with other methods of control, such as cultural strategies, would go along way in integrated stem borer management for improved food security. Understanding the genetic mechanisms underlying $B$. fusca tolerance in sorghum, is imperative for an effective breeding strategy. Estimation of broad and narrow sense heritability in diploids such as sorghum is based on total genetic and additive genetic variance (Falconer and Mackay, 1996). Combining ability is the capability of parents to combine amid each other in hybridisation so that favourable alleles are transmitted to their progenies (Panhwar et al., 2008). The concept of combining ability has been employed in other crops, particularly maize, in determining superior combiners for agronomic, disease and insect pest resistance traits. The objective of this study was to investigate the type of gene action conditioning tolerance to $B$. fusca in sorghum.

\section{MATERIALS AND METHODS}

Experimental site. Two experiments were conducted at the University of Embu, field station for two seasons in 2011/2012 (short and long rain seasons). The rainfall pattern at Embu is bi-modal, with two distinct rainy seasons. Long rains occur between March and June; while the short rains fall between October and December (Jaetzold and Schmidt, 1982). Rainfall quantity received varies with altitude, averaging about $1067.5 \mathrm{~mm}$ annually and ranging from $640 \mathrm{~mm}$ in some areas to as high as 1,495 mm per annum. Embu lies between longitude $37^{\circ} 42^{\prime} \mathrm{E}$ and latitude $0^{\circ} 44^{\prime}$ $\mathrm{S}$, at an elevation of 1,510 metres above sea level (Jaetzold and Schmidt, 1982). The site has maximum and minimum daily temperatures of 25 and $14^{\circ} \mathrm{C}$. The soils are deep, well weathered Humic Nitisols with moderate to high inherent fertility (Jaetzold and Schmidt, 1982).

Experimental materials. The experimental materials consisted of sorghum lines with varying levels of resistance to $B$. fusca (Table 1). North Calorina mating design 2 was employed, where 15 lines (Gadam, ICSA 464, ICSA 467, ICSA 472, ICSA 474, ICSB 464, ICSB 467, ICSB 474, IESV 91131 DL, IESV 93042 SH, IS 21879, IS 21881, IS 8193 , Macia and Seredo) were used as females (lines) and 2 (KARI-Mtama-1 and ICSB 473) as males (testers).

Classification of the sorghum lines into different categories was based on leaf damage, deadheart, exit holes and stem tunneling in 2010 long and short rain seasons (Muturi et al., 2012; Muturi et al., 2014). The test material was sown in an á-lattice design, consisting of sixteen plots in three blocks, replicated twice. The rows were $2 \mathrm{~m}$ long and $0.75 \mathrm{~m}$ apart, and the spacing between plants within rows was $0.25 \mathrm{~m}$. Recommended agronomic practices such as weeding and supplemental irrigation were carried out when necessary.

Stem borer neonates. First instar neonates of $B$. fusca utilised in this study were obtained from the International Centre of Insect Physiology and Ecology (ICIPE), Nairobi, Kenya. At 30 days after sowing, five plants in each row were tagged and artificially infested on the whorls with five larvae per plant using a camel hairbrush.

Data collection. Data on leaf damage and dead heart were collected per plant basis, at two and four weeks after artificial infestation. Percentages of plants showing leaf and dead heart were computed by expressing the number of plants damaged as a percentage of the total number of plants sampled. At harvest, all the leaves were removed from the tagged plants and the numbers of stem borer exit holes on the stem counted on each sampled plant. The main stem of plants infested with stem borer larvae were split open from the base to the apex using a kitchen knife; and the cumulative tunnel length measured.

Seedling vigor was scored at 30 days after sowing on a scale of $1-5$, where $1=$ low vigour (plants showing minimum growth, less 
TABLE 1. Characteristics of sorghum lines used in this study

\begin{tabular}{|c|c|c|c|}
\hline Genotype & Pedigree & Source of seed & Reaction to Busseolafusca \\
\hline ICSA 474 & (IS 18432 X ICSB 6)11-1-1-2-2 & ICRISAT India & MR \\
\hline Seredo & Seredo & ICRISAT Kenya & MR \\
\hline ICSB 474 & (IS 18432 X ICSB 6)11-1-1-2-2 & ICRISAT India & MR \\
\hline ICSA 464 & [(ICSB 11 X ICSV 702)XPS 19349B]5-1-2-2 & ICRISAT India & MR \\
\hline ICSA 472 & (ICSB 51 X ICSV 702)7-3-1 & ICRISAT India & MR \\
\hline IS 21879 & IS 21879 & ICRISAT India & MS \\
\hline *KARI-Mtama-1 & KARI-Mtama-1 & ICRISAT Kenya & MS \\
\hline ICSA 467 & [(ICSB 11 X ICSV 700)XPS 19349B]XICSB 13]4-1 & ICRISAT India & MS \\
\hline IESV $93042 \mathrm{SH}$ & IESV $93042 \mathrm{SH}$ & ICRISAT Kenya & MS \\
\hline *ICSB 473 & (ICSB 102 X ICSV 700)5-2-4-1-2 & ICRISAT India & MS \\
\hline Macia & Macia & ICRISAT Kenya & $\mathrm{R}$ \\
\hline IESV91131 DL & IESV $91131 \mathrm{DL}$ & ICRISAT Kenya & $\mathrm{R}$ \\
\hline ICSB 464 & [(ICSB 11 X ICSV 702)XPS 19349B]5-1-2-2 & ICRISAT India & $\mathrm{R}$ \\
\hline ICSB 467 & [(ICSB 11 X ICSV 700)XPS 19349B]XICSB 13]4-1 & ICRISAT India & $\mathrm{R}$ \\
\hline IS 21881 & IS 21881 & ICRISAT India & $\mathrm{R}$ \\
\hline Gadam & Gadam & ICRISAT Kenya & $\mathrm{R}$ \\
\hline IS 8193 & IS 8193 & ICRISAT India & $\mathrm{S}$ \\
\hline
\end{tabular}

$\mathrm{R}=$ Resistant, $\mathrm{MR}=$ moderately resistant, $\mathrm{MS}=$ moderately susceptiblet, $\mathrm{S}=$ susceptibe, *male parents 
leaf expansion and poor adaptation, $3=$ moderate vigor; $5=$ high vigour (tall plants with expanded leaves and robustness) (Aruna and Padmaja, 2009). Leaf glossiness was recorded at 30 days after sowing, on a scale of $1-5$ where $1=$ highly glossy, $3=$ moderately glossy, and $5=$ non glossy (Dhillon et al., 2005). Waxy bloom was recorded on a scale of $1-9$; where $1=$ no observable bloom, $3=$ slightly present, $5=$ medium, $7=$ mostly bloomy, $9=$ completely bloomy at the 50\% flowering stage (Dhillon et al., 2005).

Agronomic traits monitored included plant height, days to panicle emergence, $50 \%$ flowering and panicle length. Total grain yield and hundred-grain mass were recorded for each of the sampled plants, using a weighing balance (Mettler PM 6000, CH- 8606 GREIFENSEE-ZURICH, made in Switzerland).

Statistical analyses. Data on percentages were arcsin transformed; while those of counts were $\log$ transformed before analysis of variance to normalise the data. General analysis of variance was performed for all the traits observed using GenStat version 14 statistical software (GenStat 12 VSN 2009). Genetic analyses were performed using the Line $\mathrm{X}$ tester method using the same software (Panhwaret al., 2008).

The analysis facilitated estimate of the variances from expected mean squares and general combining ability (GCA) effects, representing additive gene effects and specific combining ability (SCA), denoting nonadditive gene effects. The sums of squares of the crosses were partitioned into GCA and SCA effects, and their interaction with the environment was estimated. Narrow-sense heritability and proportional contribution of females, males, and their interactions were also computed.

Narrow-sense heritability $=$ $(\mathrm{Vgca}) /(\mathrm{Vgca}+\mathrm{Vsca}+\mathrm{VE})\} \times 100$;

Where:
Vgca = general combining ability variance,

Vsca $=$ specific combining ability variance, and

$\mathrm{VE}=$ error variance (Dhillon et al., 2006).

Relative importance of GCA and SCA was estimated according to Baker's (1978) as:

$\left[\delta^{2} \mathrm{GCA}_{(\mathrm{f})}+\delta^{2} \mathrm{GCA}_{(\mathrm{m})}\right] /\left[\delta^{2} \mathrm{GCA}_{(\mathrm{f})}+\delta^{2} \mathrm{GCA}\right.$ $\left.(\mathrm{m})+\delta^{2} \mathrm{SCA}\right]$

Where:

$\delta^{2} \mathrm{GCA}_{(\mathrm{f})}, \delta^{2} \mathrm{GCA}_{(\mathrm{m})}$ and $\delta^{2} \mathrm{SCA}$ are the variance components for GCA and SCA, respectively.

Correlation analysis was also performed to understand the association between the morphological and traits linked with sorghum resistance to $B$. fusca.

\section{RESULTS}

\section{Plant damage and morphological traits.}

The results of plant damage and morphological traits are presented in Table 2. The mean squares due to GCAf were significant $(\mathrm{P}=<$ 0.05 or $\mathrm{P}=<0.01$ ) for leaf damage, exit holes, stem tunneling and vigour. Significant GCAm were observed on exit holes, leaf glossiness and bloom waxiness. Significant SCA mean squares were observed on exit holes and stem tunneling damages. Baker's ratio estimates for leaf feeding, deadheart, exit holes and stem tunneling damages ranged between $15-46 \%$; while that of leaf glossiness, seedling vigour and bloom waxiness ranged between $38-43 \%$. Narrow sense heritability for leaf damage, deadheart, exit holes and stem tunnels ranged from 4 to $26 \%$; while that of glossiness, vigour and bloom waxiness ranged between $38-56 \%$.

Agronomic traits. Data of plant height, panicle emergence, days to $50 \%$ flowering, panicle length, total grain weight and hundred grain mass are presented in Table 3. 
TABLE 2. Mean squares for general and specific combining ability for various damage by the African stemborer and morphological traits and heritability in sorghum

\begin{tabular}{|c|c|c|c|c|c|c|c|c|}
\hline Source of variation & d.f & $\mathrm{DH}$ & $\mathrm{LD}$ & $\mathrm{EH}$ & ST & G & $\mathrm{VG}$ & BW \\
\hline Rep & 1 & 913.7 & 2382.6 & $0.59 \mathrm{~ns}$ & 640.9 & 0.8 & 0.3 & 0.469 \\
\hline Season & 1 & $2397.3 *$ & $2207.3^{*}$ & $0.01 \mathrm{~ns}$ & 396.1ns & $0.6 \mathrm{~ns}$ & $4.9 * *$ & $7.3^{*}$ \\
\hline GCAf & 14 & $323.4 \mathrm{~ns}$ & $726.8^{*}$ & $0.13^{*}$ & $770.5^{* *}$ & $0.9 \mathrm{~ns}$ & $0.9 * *$ & $2.2 \mathrm{~ns}$ \\
\hline GCAm & 1 & $136.9 \mathrm{~ns}$ & $949.2 \mathrm{~ns}$ & $0.73 * *$ & $9.4 \mathrm{~ns}$ & $2.1 *$ & $0.7 \mathrm{~ns}$ & $21.3 * *$ \\
\hline SCA & 14 & $480.8 \mathrm{~ns}$ & $621.9 \mathrm{~ns}$ & $0.30 * *$ & $842.4 * *$ & $0.4 \mathrm{~ns}$ & $0.2 \mathrm{~ns}$ & $2.2 \mathrm{~ns}$ \\
\hline Residual & 59 & 335.6 & 368.9 & 0.07 & 151.2 & 0.5 & 0.234 & 1.494 \\
\hline \multicolumn{9}{|c|}{ Proportional contribution to total variance } \\
\hline Females & & 6.1 & 178.95 & 0.03 & 309.65 & 0.17 & 0.27 & 0.37 \\
\hline Males & & 13.25 & 38.69 & 0.04 & 9.45 & 0.1 & 0.03 & 1.32 \\
\hline Females x males & & 145.2 & 253 & 0.23 & 691.2 & 0.07 & 0.01 & 0.75 \\
\hline Baker's Ratio & & 0.15 & 0.46 & 0.25 & 0.3 & 0.79 & 0.97 & 0.69 \\
\hline \multicolumn{9}{|c|}{ Narrow sense heritability (\%) } \\
\hline & & 4 & 26 & 21 & 26 & 38 & 56 & 43 \\
\hline
\end{tabular}

*,** Data significant at $<0.05$ and $<0.01$ probability level respectively; ns = non-significant; DH $=$ Deadheart $\%$, LD $=$ Leaf damage $(\%)$, EH $=$ Exit holes, $\mathrm{S} \mathrm{T}=$ stem tunneling, $\mathrm{GL}=$ Glosiness, $\mathrm{VG}=$ vigour, $\mathrm{BL}=$ Bloom waxiness, $\mathrm{DF}=$ Degrees of freedom, GCAf = general combining ability for females, GCAm = general combining ability for males, $\mathrm{SCA}=$ specific combining ability 
TABLE 3. Mean squares for general and specific combining ability for various agronomic traits and heritability estimates in sorghum

\begin{tabular}{lrllllll}
\hline Source of variation & d.f. & PH & PE & FL & PL & TGW & HGM \\
\hline Rep stratum & 1 & 608 & 44.4 & 61.6 & 58.3 & 341.1 & 1 \\
Season & 1 & $15790.1^{* *}$ & $745.0^{* *}$ & $1540.8^{* *}$ & $2760.6^{* *}$ & $6.6 \mathrm{~ns}$ & $1.1 \mathrm{~ns}$ \\
GCAf & 14 & $8449.9^{* *}$ & $33.8^{*}$ & $34.8^{*}$ & $55.7^{* *}$ & $447.0^{* *}$ & $4.9^{* *}$ \\
GCAm & 1 & $3322.9^{*}$ & $99.0^{*}$ & $116.0^{*}$ & $19.8 \mathrm{~ns}$ & $316.3^{*}$ & $2.1^{*}$ \\
SCA & 14 & $6020.1^{* *}$ & $15.1 \mathrm{~ns}$ & $20.5 \mathrm{~ns}$ & $130.0^{* *}$ & $372.8^{* *}$ & $2.4^{* *}$ \\
Residual & 539 & 668.2 & 17.6 & 18.1 & 11.1 & 76.8 & 0.5
\end{tabular}

\section{Proportional contribution to total variance}

$\begin{array}{lllllll}\text { Females } & 3890.85 & 22.32 & 8.37 & 22.32 & 185.11 & 2.2 \\ \text { Males } & 176.98 & 0.58 & 6.53 & 0.58 & 15.96 & 0.11 \\ \text { Females X Males } & 5351.9 & 118.97 & 2.39 & 118.97 & 295.99 & 1.95 \\ \text { Baker's Ratio } & 0.43 & 0.16 & 0.86 & 0.16 & 0.4 & 0.54\end{array}$

Narrow sense heritability (\%)

$\begin{array}{llllll}40 & 15 & 42 & 15 & 35 & 49\end{array}$

$*$, ** Data significant at $<0.05$ and $<0.01$ probability level respectively; $\mathrm{ns}=$ non-significant PH $=$ Plant height, $\mathrm{PL}=$ Panicle length, $\mathrm{FL}=50 \%$ flowering, $\mathrm{PL}=$ Panicle length, TGW = Total grain weight, $\mathrm{HGM}=$ Hundred grain mass, $\mathrm{DF}=$ Degrees of freedom, $\mathrm{GCA}_{\mathrm{f}}=$ general combining ability for females, $\mathrm{GCA}_{\mathrm{m}}=$ general combining ability for males, $\mathrm{SCA}=$ specific combining ability

Mean squares due to GCAf and GCAm were significant $(\mathrm{P}<0.05)$ for plant height, panicle emergence, days to $50 \%$ flowering, total grain weight, hundred grains mass except for GCAm for panicle length. SCA mean squares were significant for plant height, panicle length, total grain weight and hundred grains mass. Baker's ratio for agronomic traits ranged between 16 - $86 \%$ while narrow sense heritability estimates ranged between 15 $49 \%$.

Stem borer damage, agronomic and morphological traits. Results for exit holes, stem tunneling, plant height, panicle length, total grain yield and hundred grain mass were significant (Table 4). Cross IESV 93042 SH X ICSB 473 suffered the least deadheart damage; while ICSB 467 X KARI-Mtama-1 suffered the most to the most damage.
Cross ICSB 464 X ICSB 473 suffered the least leaf feeding damage; while IS $21881 \mathrm{X}$ KARI-Mtama-1 suffered the most damage. ICSA 467 X ICSB 473 and ICSB 464 X ICSB 473 scored the least exit holes damage; while IS 8193 X KARI-Mtama-1 suffered the most damage. ICSB 464 X ICSB 473 had the least stem tunneling damage; while ICSB $464 \mathrm{X}$ KARI-Mtama-1 suffered the most damage. Cross ICSB 464 X ICSB 473 scored the least deadheart, leaf feeding, stem tunnels and exit holes damages combined, and also scored the highest hundred grain mass. Panicle length ranged from 17 to $26 \mathrm{~cm}$ on crosses ICSA 474 X ICSB 473 and ICSA 467 X KARIMtama-1. Cross ICSB 474 X KARI-Mtama-1 was the tallest $(154 \mathrm{~cm})$; while ICSB $464 \mathrm{X}$ ICSB 473 was the shortest $(104 \mathrm{~cm})$. Days to $50 \%$ flowering ranged from 65 - 76 days for Gadam X KARI-Mtama-1 and IS 21881 X 
TABLE 4. Damage, agronomic and morphological traits for sorghum F1 hybrids

\begin{tabular}{|c|c|c|c|c|c|c|c|c|c|c|c|c|}
\hline F1 hybrids & $\mathrm{DH}$ & LD & $\mathrm{EH}$ & ST & $\mathrm{PH}$ & FL & PL & TGW & HGM & BW & G & VG \\
\hline ICSA 467 X KARI-Mtama-1 & 35.8 & 38.9 & 0.7 & 14.0 & 128 & 72 & 26 & 38.3 & 3.1 & 6.5 & 2.8 & 3.3 \\
\hline ICSB 474 X KARI-Mtama-1 & 13.3 & 16.4 & 0.8 & 19.3 & 153 & 71 & 23 & 31.0 & 2.9 & 6.8 & 2.9 & 3.5 \\
\hline IESV 91131 DL X KARI-Mtama-1 & 19.6 & 54.5 & 0.7 & 17.8 & 112 & 71 & 24 & 30.2 & 3.1 & 4.5 & 3.4 & 3.4 \\
\hline ICSA 472 X ICSB 473 & 26.0 & 26.3 & 0.7 & 15.6 & 137 & 75 & 17 & 29.1 & 3.1 & 5.5 & 3.5 & 3.4 \\
\hline ICSB 467 X KARI-Mtama-1 & 51.1 & 45.0 & 0.7 & 20.5 & 125 & 71 & 23 & 26.9 & 3.1 & 6.0 & 2.9 & 3.1 \\
\hline ICSB 464 X ICSB 473 & 16.4 & 15.9 & 0.4 & 9.1 & 104 & 72 & 18 & 25.6 & 4.0 & 7.0 & 2.8 & 3.3 \\
\hline ICSA 472 X KARI-Mtama-1 & 32.3 & 48.8 & 0.5 & 12.6 & 145 & 73 & 21 & 24.4 & 2.7 & 6.0 & 2.7 & 2.9 \\
\hline ICSA 464 X KARI-Mtama-1 & 23.1 & 48.5 & 0.6 & 13.3 & 120 & 71 & 24 & 24.3 & 2.8 & 5.8 & 3.0 & 2.9 \\
\hline ICSA 474 X ICSB 473 & 26.3 & 44.4 & 0.6 & 16.7 & 119 & 72 & 17 & 23.9 & 2.9 & 7.0 & 2.3 & 3.8 \\
\hline IESV 91131 DL X ICSB 473 & 26.3 & 48.2 & 0.7 & 27.7 & 106 & 72 & 19 & 23.6 & 2.9 & 7.0 & 3.0 & 3.4 \\
\hline ICSB 474 X ICSB 473 & 38.9 & 26.3 & 0.7 & 19.2 & 145 & 71 & 18 & 23.1 & 2.7 & 6.8 & 2.9 & 4.0 \\
\hline ICSA 467 X ICSB 473 & 35.2 & 38.7 & 0.4 & 10.9 & 120 & 72 & 20 & 22.9 & 2.7 & 7.0 & 2.3 & 3.0 \\
\hline IS 21879 X ICSB 473 & 25.7 & 32.6 & 0.6 & 28.6 & 121 & 75 & 19 & 22.9 & 2.3 & 7.0 & 2.3 & 3.4 \\
\hline IS 21881 X KARI-Mtama-1 & 32.0 & 64.6 & 0.8 & 20.0 & 115 & 71 & 24 & 22.8 & 2.6 & 6.0 & 3.4 & 2.4 \\
\hline Gadam X KARI-Mtama-1 & 22.5 & 28.1 & 0.7 & 13.3 & 118 & 65 & 22 & 22.2 & 2.9 & 5.5 & 3.3 & 3.0 \\
\hline ICSA 464 X ICSB 473 & 24.8 & 17.9 & 0.6 & 9.3 & 112 & 72 & 20 & 21.9 & 3.1 & 6.3 & 2.3 & 3.4 \\
\hline IS 21879 X KARI-Mtama-1 & 32.6 & 44.4 & 0.7 & 19.7 & 129 & 76 & 22 & 21.5 & 2.2 & 6.5 & 2.9 & 3.0 \\
\hline IESV 93042 SH X KARI-Mtama-1 & 32.0 & 42.4 & 0.6 & 14.0 & 138 & 72 & 23 & 21.5 & 2.5 & 6.1 & 3.6 & 3.1 \\
\hline Gadam X ICSB 473 & 16.4 & 35.5 & 0.7 & 15.6 & 110 & 68 & 18 & 21.3 & 2.9 & 7.0 & 3.5 & 2.3 \\
\hline IESV 93042 SH X ICSB 473 & 9.8 & 54.8 & 0.7 & 23.6 & 130 & 72 & 18 & 21.3 & 2.8 & 5.5 & 3.1 & 3.3 \\
\hline Seredo X ICSB 473 & 35.2 & 26.3 & 0.7 & 27.7 & 120 & 69 & 20 & 20.8 & 2.4 & 6.5 & 3.0 & 3.1 \\
\hline IS 8193 X ICSB 473 & 35.8 & 38.4 & 0.8 & 22.3 & 129 & 69 & 20 & 20.5 & 2.2 & 7.0 & 2.9 & 3.4 \\
\hline Macia X ICSB 473 & 42.1 & 36.1 & 0.7 & 24.0 & 111 & 70 & 19 & 20.4 & 2.9 & 6.5 & 3.1 & 3.2 \\
\hline IS 21881 X ICSB 473 & 19.3 & 19.3 & 0.6 & 19.5 & 107 & 76 & 20 & 19.9 & 2.4 & 6.3 & 3.4 & 2.7 \\
\hline Seredo X KARI-Mtama-1 & 47.9 & 26.0 & 0.8 & 22.2 & 128 & 67 & 25 & 19.7 & 2.8 & 4.0 & 3.1 & 3.0 \\
\hline ICSB 464 X KARI-Mtama-1 & 35.2 & 16.4 & 0.8 & 36.9 & 112 & 71 & 22 & 19.5 & 2.5 & 6.5 & 3.1 & 3.4 \\
\hline ICSA 474 X KARI-Mtama-1 & 19.6 & 41.8 & 0.8 & 22.4 & 127 & 71 & 22 & 19.4 & 2.9 & 7.0 & 2.8 & 3.5 \\
\hline Macia X KARI-Mtama-1 & 19.9 & 32.3 & 0.7 & 19.1 & 119 & 71 & 24 & 19.4 & 2.7 & 4.8 & 3.3 & 3.1 \\
\hline
\end{tabular}


ICSB 473, respectively. Cross ICSA 467 X KARI-Mtama-1 produced about three times more grain weight than IS 8193 X KARIMtama-1, which produced the least. Hundred grain mass ranged from 1.4 to $4.0 \mathrm{~g}$ on IS 8193 X KARI-Mtama- 1 and ICSB 464 X ICSB 473 , respectively.

Gene action. Results for leaf damage, deadheart, exit holes and stem tunnels GCA effects are shown in Table 5. Maximum GCA effects for leaf damage, deadheart and stem damages were displayed by genotypes Gadam, ICSB 464 and ICSA 464, respectively. Generally, ICSB 473 (male) contributed favourable alleles for resistance to $B$. fusca. Seredo scored the highest positive GCA effects on dead heart, stem exit holes and stem tunneling damages. IESV 91131DL showed the highest undesirable GCA effect towards leaf feeding damage. Maximum GCA effect for plant height was observed on ICSB 474; while Gadam scored the maximum negative GCA effects for plant height (Table 5). Highest positive GCA effects for days to $50 \%$ flowering among females were recorded on IS 21879 and IS 21881; while a negative maximum GCA effect was recorded on Gadam. High positive significant GCA effect for total grain yield was observed on ICSA 467 among lines and KARI-Mtama- 1 among the males. A high GCA effects for bloom waxiness were observed on ICSA 474, ICSA 467 and ICSB 474. Seedlings of ICSA 474 and ICSA 467 were highly vigorous and glossy; while for IS 8193 and IS 21881, they were non-glossy and the least vigorous.

Results on specific combining ability for the different damages, agronomic and morphological characteristics are presented in Table 6. There were significant differences in SCA effects among crosses with regard to leaf damage, exit holes, stem tunnels, plant height, panicle length, grain yield and leaf glossiness. Most of the other SCA estimates were negative, low or not significant. Crosses that showed the least negative SCA effects for deadheart, leaf and stem damages were ICSB 
TABLE 5. General combining ability (GCA) effects of females and males for damage, agronomic and morphological traits in sorghum

\begin{tabular}{|c|c|c|c|c|c|c|c|c|c|c|c|c|}
\hline Females & DH & LD & $\mathrm{EH}$ & ST & $\mathrm{PH}$ & FL & PL & TGW & HGM & BW & GL & VG \\
\hline Gadam & -8.50 & -3.30 & 0.03 & -4.95 & -21.90 & -3.53 & -1.45 & -1.28 & 0.15 & 0.07 & 0.38 & -0.56 \\
\hline ICSA 464 & -4.10 & -1.90 & -0.08 & -8.13 & -5.80 & 0.47 & -0.37 & 0.02 & 0.20 & -0.18 & -0.36 & -0.03 \\
\hline ICSA 467 & 7.50 & 3.80 & -0.08 & -6.98 & -5.30 & 2.47 & -0.02 & $7.56^{* *}$ & 0.14 & 0.57 & -0.45 & -0.04 \\
\hline ICSA 472 & 1.10 & 2.50 & -0.06 & -5.28 & -16.10 & -1.65 & 0.33 & $3.72 *$ & 0.17 & -0.43 & 0.10 & -0.03 \\
\hline ICSA 474 & -5.10 & 8.10 & 0.02 & 0.15 & $26.50 *$ & 2.22 & -1.00 & -1.37 & 0.15 & $0.82 *$ & -0.50 & $0.47 *$ \\
\hline ICSB 464 & -2.20 & -18.90 & -0.06 & 3.58 & $14.50 *$ & -1.90 & $2.10 *$ & -0.46 & $0.53 * *$ & 0.57 & -0.06 & 0.16 \\
\hline ICSB 467 & 10.50 & -1.00 & -0.04 & 2.45 & 6.40 & 0.47 & $1.82 *$ & -0.80 & 0.13 & 0.20 & -0.18 & -0.03 \\
\hline ICSB 474 & -1.90 & -13.70 & 0.05 & -0.15 & $28.80^{*}$ & -2.53 & 0.88 & $4.03^{*}$ & 0.12 & 0.57 & -0.12 & $0.60 *$ \\
\hline IESV 91131 DL & -5.10 & $16.30 *$ & 0.02 & 3.35 & 5.80 & -3.15 & 0.88 & $3.83^{*}$ & $0.26^{*}$ & -0.43 & 0.19 & 0.22 \\
\hline IESV $93042 \mathrm{SH}$ & -7.10 & 13.60 & -0.01 & -0.60 & -13.40 & 1.85 & -0.15 & -1.65 & -0.09 & -0.37 & 0.38 & 0.03 \\
\hline IS 21879 & 1.10 & 3.50 & -0.02 & $4.75^{*}$ & -16.50 & 3.10 & -0.72 & -0.82 & -0.49 & 0.57 & -0.43 & 0.03 \\
\hline IS 21881 & -2.30 & 6.90 & 0.03 & 0.36 & 1.60 & 0.47 & 0.20 & -1.70 & -0.26 & -0.05 & 0.38 & -0.62 \\
\hline IS 8193 & -0.50 & -6.10 & $0.13^{*}$ & 3.75 & 7.20 & 0.22 & 0.70 & -5.12 & -0.92 & -0.43 & 0.38 & -0.09 \\
\hline Macia & 3.00 & -0.90 & 0.03 & 2.15 & -13.30 & 0.60 & -2.20 & -3.12 & 0.07 & -0.55 & 0.23 & 0.01 \\
\hline Seredo & $13.50^{*}$ & -8.90 & 0.05 & $5.55^{*}$ & $11.60^{*}$ & 0.85 & -0.97 & -2.83 & -0.16 & -0.93 & 0.07 & -0.12 \\
\hline \multicolumn{13}{|l|}{ Males } \\
\hline ICSB 473 & -1.05 & -2.75 & -0.03 & 0.12 & 2.37 & -0.98 & 0.18 & -0.72 & 0.06 & $0.42 *$ & -0.13 & 0.08 \\
\hline KARI-Mtama-1 & 1.08 & 2.85 & 0.04 & -0.13 & -2.34 & 0.98 & -0.19 & 0.73 & -0.06 & -0.42 & 0.13 & -0.08 \\
\hline
\end{tabular}

*,** Data significant at $<0.05$ and $<0.01$ probability level respectively; $\mathrm{DH}=$ Dead heart $\%, \mathrm{LD}=\mathrm{Leaf}$ damage $(\%), \mathrm{EH}=\mathrm{Exit}$ holes, $\mathrm{ST}=$ stem tunneling, $\mathrm{PH}=$ Plant height, $\mathrm{FL}=50 \%$ flowering, $\mathrm{PL}=$ Panicle length, $\mathrm{TGW}=$ Total grain weight, $\mathrm{HGM}=$ Hundred grain mass, $\mathrm{BL}=\mathrm{Bloom}$ waxiness, $\mathrm{GL}=\mathrm{Glosiness}$, $\mathrm{VG}=$ vigour 
TABLE 6. Specific combining ability estimates of F1 hybrids from line $\mathrm{x}$ tester analysis of various characters in sorghum

\begin{tabular}{|c|c|c|c|c|c|c|c|c|c|c|c|c|}
\hline F1 hybrids & $\mathrm{DH}$ & $\mathrm{LD}$ & $\mathrm{EH}$ & ST & $\mathrm{PH}$ & FL & PL & TGW & HGM & $\mathrm{BW}$ & GL & VG \\
\hline Gadam X ICSB 473 & 6.29 & 7.71 & 0.01 & $6.86^{*}$ & 0.15 & $5.40 * *$ & $-1.23^{*}$ & 1.97 & -0.31 & 0.27 & 0.26 & -0.01 \\
\hline Gadam X KARI-Mtama-1 & 10.84 & -1.21 & -0.07 & $3.78^{*}$ & 19.72 & 7.33 & 3.60 & 0.61 & 2.36 & -0.39 & $-0.49 *$ & 0.88 \\
\hline ICSA 464 X ICSB 473 & 3.99 & 17.91 & -0.09 & $6.86^{*}$ & 11.15 & -0.60 & -0.03 & -0.13 & $-0.21 *$ & -0.03 & 0.56 & 0.19 \\
\hline ICSA 464 X KARI-Mtama-1 & 5.69 & $-14.21 *$ & 0.03 & 10.08 & -6.68 & 2.33 & 0.30 & 0.01 & 2.16 & 0.31 & 0.71 & $-0.27 *$ \\
\hline ICSA 467 X ICSB 473 & $-13.21 *$ & -3.09 & 0.01 & 6.16 & -11.35 & -1.60 & -0.93 & -29.61 & -0.51 & -0.73 & $0.76^{*}$ & $-0.31 *$ \\
\hline ICSA 467 X KARI-Mtama-1 & $-7.96^{*}$ & -4.41 & $0.23 * *$ & 8.48 & 14.92 & $-4.67 *$ & 0.40 & -0.99 & 2.56 & -0.39 & 0.71 & $-0.22 *$ \\
\hline ICSA 472 X ICSB 473 & -3.51 & $-12.99 *$ & 0.21 & $7.56^{*}$ & 19.05 & $-2.60 *$ & 1.57 & -0.23 & -0.11 & -0.23 & 0.06 & 0.09 \\
\hline ICSA 472 X KARI-Mtama-1 & 1.24 & 7.99 & -0.07 & 3.78 & 6.02 & 2.33 & -2.80 & $-7.19 *$ & 2.16 & 1.11 & $0.31 *$ & $-0.23 *$ \\
\hline ICSA 474 X ICSB 473 & 9.19 & -5.99 & -0.14 & -2.24 & -8.25 & 0.40 & -0.53 & $4.77 *$ & $-0.31 *$ & -1.23 & $0.76^{*}$ & $-0.51^{*}$ \\
\hline ICSA 474 X KARI-Mtama-1 & 7.64 & -10.11 & $0.08 *$ & $2.68 *$ & -27.78 & -2.67 & $2.00^{*}$ & -1.99 & 2.36 & -0.39 & $0.71 *$ & $-0.62 *$ \\
\hline ICSB 464 X ICSB 473 & -6.41 & 19.41 & $-0.19 *$ & -16.74 & -29.85 & 0.40 & -5.83 & -1.43 & 0.09 & -0.73 & $0.46^{*}$ & $-0.41^{*}$ \\
\hline ICSB 464 X KARI-Mtama-1 & 10.84 & 18.39 & 0.23 & 10.28 & -30.08 & 0.33 & 1.10 & 2.41 & 1.26 & -0.39 & 0.21 & -0.12 \\
\hline ICSB 467 X ICSB 473 & $-22.31 *$ & $-9.19 *$ & 0.01 & -0.34 & -0.15 & -1.60 & -3.33 & $-2.73^{*}$ & $-0.51 *$ & -0.23 & $0.66^{*}$ & -0.11 \\
\hline ICSB 467 X KARI-Mtama-1 & 1.24 & 11.19 & 0.03 & $-3.72 *$ & -19.78 & 1.33 & -0.90 & 4.31 & 2.56 & -0.19 & 0.21 & 0.08 \\
\hline ICSB 474 X ICSB 473 & $15.49 *$ & 19.41 & -0.09 & 0.86 & -39.55 & 1.40 & -2.13 & -6.83 & $-0.31 *$ & $-1.03 *$ & $0.66^{*}$ & $-0.51 *$ \\
\hline ICSB 474 X KARI-Mtama-1 & -11.66 & 7.99 & -0.07 & 0.18 & $-5.18 *$ & $1.33^{*}$ & -0.10 & -1.19 & 2.56 & -0.19 & 0.11 & -0.82 \\
\hline IESV 91131 DL X ICSB 473 & 9.19 & -18.69 & 0.01 & $2.36^{*}$ & -11.15 & 3.40 & -0.73 & -6.03 & -0.51 & -5.36 & 0.16 & -0.41 \\
\hline IESV 91131 DL X KARI-Mtama-1 & 0.94 & $-13.91 *$ & -0.07 & -8.32 & -7.38 & 2.33 & -1.50 & $-1.69 *$ & 2.36 & -0.39 & 0.01 & $-0.27 *$ \\
\hline IESV 93042 SH X ICSB 473 & -3.21 & -6.59 & 0.11 & 6.16 & -3.05 & 3.40 & -0.53 & $2.67 *$ & 0.09 & -0.33 & -0.04 & -0.11 \\
\hline IESV 93042 SH X KARI-Mtama-1 & -4.76 & -20.51 & 0.01 & $-3.62 *$ & 22.82 & 0.33 & 0.30 & 0.61 & 2.46 & 1.11 & -0.09 & -0.12 \\
\hline IS 21879 X ICSB 473 & -3.81 & $-8.59 *$ & 0.01 & 0.46 & 11.15 & -13.60 & 1.17 & $2.67 *$ & $0.39 *$ & -0.73 & $0.66^{*}$ & -0.01 \\
\hline IS 21879 X KARI-Mtama-1 & -5.36 & 1.69 & 0.07 & -9.22 & $14.72 *$ & -16.67 & -0.20 & -0.99 & 2.96 & -12.63 & 0.71 & $-0.22 *$ \\
\hline IS 21881 X ICSB 473 & -3.21 & $-28.79 *$ & -0.06 & 0.16 & -23.65 & 3.40 & -1.03 & 1.37 & 0.19 & -0.23 & 0.16 & 0.59 \\
\hline IS 21881 X KARI-Mtama-1 & -4.76 & 14.99 & 0.03 & -0.12 & 13.32 & 2.33 & 0.10 & 2.01 & 2.66 & 0.31 & -0.39 & 0.48 \\
\hline IS 8193 X ICSB 473 & 9.49 & 16.21 & -0.09 & $-3.84 *$ & 10.45 & 2.40 & 2.07 & 8.77 & 1.19 & $1.27 *$ & $-0.34 *$ & 0.19 \\
\hline IS 8193 X KARI-Mtama-1 & $7.94^{*}$ & -4.11 & $-0.17 *$ & -4.62 & $-31.88 * *$ & 2.33 & -4.00 & 1.41 & 3.06 & -0.39 & 0.11 & $-0.22 *$ \\
\hline Macia X ICSB 473 & $11.87^{*}$ & 3.51 & 0.01 & 1.06 & 15.75 & -1.60 & 2.67 & $4.77 *$ & -0.11 & 0.97 & 0.26 & -0.11 \\
\hline
\end{tabular}


P.W. MUTURI et al.

467 X ICSB 473, IS 21881 X ICSB 473 and ICSB 464 X ICSB 473, respectively. Cross ICSA 467 X KARI-Mtama-1 was the best specific combiner for panicle length and total grain yield. Crosses ICSA 474 X ICSB 473 and ICSB 474 X ICSB 473recorded the highest seedling vigour. Cross ICSB 464 X ICSB 473 showed the highest hundred grain mass and was resistant to stem tunneling and exit holes damage.

Association between B. fusca damage parameters, agronomic and morphological traits. Results for correlation between damage parameters, agronomic and morphological traits are presented in Table 7.

Bloom waxiness was significantly and positively correlated with days to $50 \%$ flowering, and days to panicle emergence $(\mathrm{r}=$ $0.38^{*}$ and $\mathrm{r}=0.35^{*}$, respectively). Days to $50 \%$ flowering correlated positively and highly significantly with days to panicle emergence $\left(r=0.96^{* *}\right)$. Plant height significantly and positively correlated with vigour $(r=0.49 * *)$ and panicle length $\left(r=0.51^{* *}\right)$. A positive and significant association was observed between panicle length and vigour $\left(r=0.36^{*}\right)$. Exit holes positively and significantly correlated with stem tunnels $\left(\mathrm{r}=0.61^{* *}\right)$, and with non-glossy leaves $\left(r=0.49^{* *}\right)$. A positive and highly significant relationship was observed between dry panicle weight and total grains $(\mathrm{r}=0.94 * *)$ and with hundred grain mass $(\mathrm{r}=0.52 * *)$. Hundred grain mass positively and significantly correlated with total grain yield $\left(\mathrm{r}=0.54^{* *}\right)$.

Bloom waxiness negatively and significantly correlated with leaf glossiness $(r=-57 * *)$. Days to $50 \%$ flowering negatively and significantly correlated with leaf glossiness ( $\mathrm{r}$ $\left.=-0.70^{* *}\right)$. Leaf glossiness was negatively and highly significantly associated with days to panicle emergence $\left(\mathrm{r}=-0.63^{* *}\right)$ and vigour $\left(\mathrm{r}=-0.46^{* *}\right)$. A negative relationship was observed between exit holes and hundred grain mass $\left(r=-0.40^{*}\right)$ and exit holes and days to $50 \%$ flowering $\left(r=-0.36^{*}\right)$. A negative and highly significant association was observed between dry panicle weight and stem tunnels 
TABLE 7. Correlation matrix between damage parameters, agronomic and morphological characters in sorghum

\begin{tabular}{|c|c|c|c|c|c|c|c|c|c|c|c|c|c|c|}
\hline & $\mathrm{BW}$ & $\mathrm{DH}$ & DPW & $\mathrm{EH}$ & FL & GL & HGM & $\mathrm{LD}$ & PE & $\mathrm{PH}$ & PL & ST & TGW & VG \\
\hline BW & - & & & & & & & & & & & & & \\
\hline DH & 0.01 & - & & & & & & & & & & & & \\
\hline DPW & 0.03 & 0.09 & - & & & & & & & & & & & \\
\hline $\mathrm{EH}$ & -0.31 & 0.08 & -0.06 & - & & & & & & & & & & \\
\hline FL & $0.38 *$ & 0.1 & -0.02 & $-0.36^{*}$ & - & & & & & & & & & \\
\hline G & $-0.57 * *$ & -0.16 & -0.22 & $0.49 * *$ & $-0.70^{* *}$ & - & & & & & & & & \\
\hline HGM & 0.17 & -0.09 & $0.52 * *$ & $-0.40^{*}$ & -0.09 & -0.29 & - & & & & & & & \\
\hline LD & -0.06 & 0.09 & 0.16 & 0.05 & -0.06 & 0.02 & 0.01 & - & & & & & & \\
\hline $\mathrm{PE}$ & $0.35^{*}$ & 0.08 & 0.0 & -0.3 & $0.96^{* *}$ & $-0.63^{* *}$ & -0.15 & 0.08 & - & & & & & \\
\hline $\mathrm{PH}$ & 0.32 & 0.14 & 0.17 & 0.06 & -0.08 & -0.28 & 0.25 & -0.26 & -0.15 & - & & & & \\
\hline PL & 0.33 & 0.18 & 0.15 & 0.19 & 0.0 & -0.07 & 0.03 & -0.09 & -0.07 & $0.51 * *$ & - & & & \\
\hline ST & 0.03 & 0.17 & $-0.36^{*}$ & $0.61 * *$ & 0.11 & 0.14 & $-0.46^{* *}$ & -0.08 & 0.07 & 0.18 & $0.39 *$ & - & & \\
\hline TGW & 0.08 & 0.02 & $0.94 * *$ & -0.08 & 0.01 & -0.18 & $0.54 * *$ & 0.18 & 0.02 & 0.17 & 0.22 & $-0.38^{*}$ & - & \\
\hline $\mathrm{VG}$ & 0.25 & 0.07 & 0.25 & -0.03 & 0.32 & $-0.46^{* *}$ & 0.26 & -0.18 & 0.26 & $0.49 * *$ & $0.36^{*}$ & 0.15 & 0.25 & - \\
\hline
\end{tabular}

* ** Data significant at $<0.05$ and $<0.01$ probability level, respectively; $\mathrm{BW}=$ bloom waxiness, $\mathrm{DH}=$ Deadheart damage, $\mathrm{DPW}=\mathrm{dry}$ panicle weight, $\mathrm{EH}$ $=$ exit holes, $\mathrm{FL}=$ days to flowering, $\mathrm{GL}=$ leaf glossiness, $\mathrm{HGM}=$ hundred grain mass, $\mathrm{LD}=$ leaf damage, $\mathrm{PE}=$ panicle emrgence, $\mathrm{PH}=$ plant height, $\mathrm{PL}$

$=$ panicle length, $\mathrm{ST}=$ stem tunneling, $\mathrm{TGW}=$ total grain weight, $\mathrm{VG}=$ seedling vigour 
$(r=-0.36 *)$. A negative relationship was also observed between hundred grain mass and stem tunnels $\left(\mathrm{r}=-0.46^{* *}\right)$. Stem tunneling negatively and significantly correlated with total grain yield $\left(r=-0.38^{*}\right)$.

\section{DISCUSSION}

General combining ability (GCA), as well as specific combining ability (SCA) were significant (Tables 2 and 3), suggesting that additive and non-additive gene effects were important in conditioning resistance traits to B. fusca. The negative GCA effects for damage (leaf damage, deadheart, stem tunnels and exit holes) signified contribution of the genotype towards resistance; while positive GCA effects on the parents suggested that the those parents contributed towards B. fusca damage susceptibility in the crosses. The significance of GCAf mean squares for leaf damage, exit holes, stem tunneling, seedling vigour; and significant GCAm for exit holes, seedling vigoUr and bloom waxiness implied presence of additive genes in controlling these traits. The significance of GCAf and GCAm in controlling exit holes implies that both female and males influenced the trait. GCA (female) contribution was predominant over GCA (male) for leaf damage and stem tunneling, suggesting that that there might be maternal effects. Baker's ratio estimates indicated that leaf damage was conditioned by both additive and non-additive genes since GCA SCA ratio was almost 1:1. Thus, the response of hybrids to leaf damage could be predicted based on the GCA of the parents. This observation is supported by Aruna and Padmaja (2009) who reported that the potentiality of parents to produce better offspring with a pool of superior genes in regard to sorghum shoot damage by shoot insect pests is based on their GCA effects.

The significance of SCA over GCA as estimated from Baker's ratio for deadheart, exit holes and stem tunnels damage suggested that these characters were controlled largely by non-additive type of gene action (Table 2).
Both additive and non-additive genes conditioned plant height, panicle weight and grain yield in the current study. A study conducted by Sharma et al. (2007) on inheritance of resistance to spotted stem borer in sorghum reported that these traits were mainly controlled by additive type of gene action. The significance of SCA variance implies that cross combinations would be efficient in breeding for deadheart, exit holes and deadheart. Also, the observation suggests that inheritance depended on the resistance and susceptibility levels of the parents used in the cross. This also indicates that the GCA alone could not give a complete prediction of hybrids' deadheart and stem tolerance to $B$. fusca.

This observation is supported by findings on inheritance of resistance in maize to $B$. fusca in South Africa, that estimates of combining ability show greater SCA than GCA in most crosses (Taylor et al., 2003). Moreover, a study conducted by Andre et al. (2003) on inheritance of resistance in maize to $B$. fusca revealed that SCA was greater than GCA in most crosses. Gene effects for B. fusca resistance in sorghum are not fixable, owing to the presence of significant non-heritable interaction present in $F_{1}$. In order to fix the genes of interest, unconventional methods such as DNA marker technology can be considered to fix the genes.

Narrow sense heritability estimates for leaf damage, exit holes and stem tunneling were moderate (ranged between 21-26\%), implying that genetic gain is likely to be realised in selection. The moderate heritability estimates for leaf glossiness, seedling vigour and bloom waxiness imply that genetic gain is likely to be realised when selection is done. Genetic gain may not be realised for deadheart as estimated from the narrow sense heritability, since the values are too low. There is need to consider other options such as DNA marker technology to fix the desired genes. Further analysis using Baker's ratio equation to estimate the preponderance mode of gene action, suggested days to $50 \%$ flowering was largely conditioned 
by additive genes. Additive genes conditioned plant height, panicle weight and grain yield. A study conducted by Mohammed (2015) on quantitative genetic analysis of agronomic and morphological traits in sorghum reported that plant height and grain yield is controlled by both additive and non-additive type of gene action. Muturi et al. (2019) reported that plant height and yield related traits are controlled by both additive and non-additive types of gene action.

Parents such as IESV 91131 DL and KARIMtama-1, which scored high positive GCA effect with regard to leaf damage, deadheart, exit holes and several parameters, might have contributed alleles for susceptibility to borer damage in the crosses (Table 5). The maximum negative GCA effects observed on some parents, such as ICSB 464 and ICSB 473 , implied that these parents contributed positive alleles in the crosses in which they were involved with genes that could augment resistance to B. fusca damage. The low negative SCA effects observed on some crosses, such as ICSB 464 X ICSB 473 , implied this cross was among the best specific combinations for stem borer damage. Sharma et al. (2007) who studied inheritance to spotted stem borer in sorghum reported that parents possessing significant SCA effects for two or more resistance traits for either or more parents have good potential for use as breeding stock in the development of sorghum hybrids resistant to stem borer.

The negative relationship observed between stem tunnels, exit holes, total grain yield and hundred grain mass (Table 7) implies that stem damage contributed to significant reduction in grain yield. This observation may imply that selection for reduction in stem tunneling would lead to increased sorghum grain yield. The probable explanation could be due to the fact that stem tunneling causes obliteration of vascular tissues that are important in translocation of nutrients and water from the soil to the photosynthetic sites and from the source to the sink organs. This explanation is supported by Kfir et al. (2002) who reported that stem tunneling and feeding by stem borer destroys the growing point of sorghum, causes early leaf aging thus interfering with translocation of metabolites and nutrients that result in malformation of the grain and results to direct damage to ears in maize plant. The reverse relationship observed between hundred grain mass with exit holes and stem tunnels, implies that stem damage contributed to significant reduction in grain yield. It is worth noting that among the stem borer damage traits, stem tunneling and exit holes damage contributed to lowering of grain yield. This suggests that the two traits were key in demonstrating host resistance to B. fusca damage. A study conducted by Odiyi et al. (2007) against the effect of Sesamia calamistis (Hampson) and Eldana saccharina (Walker) in maize observed that stem tunneling was the major trait contributing to yield loss. Sorghum genotypes with highly glossy, non-vigorous seedlings suffered lower damage compaired to the non-glossy but highly vigorous ones. This may be attributed to the fact that the highly glossy, shinny leaf surfaces were not ideal for the attachment of the stemborer neonates and their habitation due to high levels of cuticular wax accumulation. Similar observations have been reported for sorghum shootfly, using some other populations, where highly glossy lines were resistant to shoot fly attack (Dhillon et al., 2005).

Tall plants were observed to have high vigour and produced long panicles (Table 7). This implies that tall plants had photosynthetic advantage and were efficient in channeling assimilates to the sink organs hence higher grain yields from such genotypes. Songa et al. (2001) reported that maize plants with many leaves $(>10)$, taller $(>150 \mathrm{~cm})$ with big stem diameter $(>2.1 \mathrm{~cm})$ had significantly increased yield. The positive and significant relationship between exit holes and stem tunnels, suggests a close and direct relationship between these two damage traits and, therefore, one of them could be used to predict the other. Moreover, 
either of the two stem damage traits can be used as B. fusca resistance trait thus reduction in phenotyping costs in terms of funds, labour and time involved in data collection.

This study also showed that the two stem damage traits i.e stem tunneling and exit hole damages are controlled by non-additive type of gene action. An inverse association was observed between bloom waxiness and leaf glossiness in the population under study. This suggests that the two resistance traits are conditioned by different alleles. Generally, there was no significant correlation between bloom waxiness and stem tunneling; bloom waxiness and deadheart and this may suggest that there are other factors of resistance at work in the population under study and those factors were not assessed in this study.

\section{ACKNOWLEDGEMENT}

Financial support was obtained from the Regional Universities Forum for Capacity Building in Agriculture (RUFORUM), Carnegie Corporation of New York, USA and the Harnessing Opportunities for Productivity Enhancement (HOPE) of Sorghum and Millets in Sub-Saharan Africa and South Asia Project funded by Bill and Melinda Gates foundation implemented by ICRISAT. Kenya Agricultural \& Livestock Research Organization (KARLO), Kiboko Research Station is thanked for hosting the research.

\section{REFERENCES}

Amsalu, D., Bekele, J. and Melaku, W. 2008. Effect of fertilizer and crop variety on the survival of Busseola fusca (Lepidoptera: Noctuidae) in cereals in Ethiopia. International Journal of Tropical Insect Science 27(3-4):172 -182.

André, A.M. van Rensburg J.B.J. and Labuschagne, M.T. 2003. Inheritance of resistance in maize to the African stalk borer, Busseola fusca (Fuller) (Lepidoptera:
Noctuidae). South African Journal of Plant and Soil 20(2):64 - 71.

Aruna, C. and Padmaja, P.G. 2009. Evaluation of genetic potential of shoot fly resistant sources in sorghum (Sorghum bicolor (L.) Moench). The Journal of Agricultural Science 147 (01):71 - 80.

Baker, R.J. 1978. Issues in diallel analysis. Crop Science 18 (4):533 - 536.

Chabi-Olaye, A., Nolte, C., Schulthess, F. and Borgemeister, C. 2005. Relationships of intercropped maize, stem borer damage to maize yield and land-use efficiency in the humid forest of Cameroon. Bulletin of Entomological Research 95(5):417 - 427.

Dhillon, M. K., Sharma, H. C. Reddy, H.C., Ram Singh, B.V.S. and Naresh, J.S. 2006. Inheritance of resistance to sorghum shoot fly. Crop Science Journal 46(3):1377 1383.

Dhillon, M.K., Sharma, H.C., Singh, R.A.M. and Naresh, J.S. 2005. Mechanisms of resistance to shoot fly, Atherigona soccata in sorghum. Euphytica 144 (3):301 - 312.

Falconer, D.S. and T.F.C. Mackay, T.F.C. 1996. Introduction to quantitative genetics. Longman, Essex, England.

GenStat, 2015. GenStat for Windows 18th Edition. VSN International, Hemel Hempstead, UK. Web page: Genstat.co.uk Jaetzold, R. and Schmidt, H. 1982. Farm Management Handbook of Kenya. Natural conditions and farm management information. Part A, B and C. Ministry of Agriculture, Kenya.

Kfir, R., Overholt, W.A., Khan, Z.R. and Polaszek, A. 2002. Biology and management of economically important lepidopteran cereal stem borers in Africa. Annual Review of Entomology 47:701 731.

Markus, S. and Gurling, B. 2006. Risk assessment for transgenic sorghum in Africa: crop-to-crop gene flow in Sorghum bicolor (L.) Moench. Crop Science Journal 46(2):790 - 798. 
Mohammed Riyazaddin, Are Ashok K., Bhavanasi Ramaiah, Munghate Rajendra S., Kavi Kishor Polavarapu, B. and Sharma Hari, C. 2015. Quantitative genetic analysis of agronomic and morphological traits in sorghum. Frontiers in Plant Science 6:945

Muhammad, A., Zahid, N., Muhammad, H.B. and Bilal, S.K. 2009. Analysis of host plant resistance in some genotypes of maize against Chilo partellus. Pakistan Journal of Botany 41(1):421 - 428.

Muturi, P.W., Rubaihayo. P., Mgonja, M., Kyamanywa, S., Sharma, H.C. and Hash, C.T. 2012. Novel source of sorghum tolerance to the African stem borer, Busseola fusca. African Journal of Plant Science 6(11):295 - 302.

Muturi, P.W., Mgonja, M. and Rubaihayo, P. 2014. Identification of new sorghum genotypes resistant to the African and spotted stem borers. International Journal of Tropical Insect Science 34(4):260 - 268.

Muturi, P.W., Mgonja, M. and Rubaihayo, P. 2019. Gene action conditioning resistance traits to spotted stem borer, Chilo partellus, in grain sorghum. International Journal of Tropical Insect Science 9: 147. https://doi.org/10.1007/s42690-01900020-x

Nyquist, W.E. and Baker, R.J. 1991. Estimation of heritability and prediction of selection response. Critical Reviews in Plant Sciences 10(3):235 - 322.

Odiyi, A.C. 2007. Relationship between stem borer resistance traits and grain yield reduction in maize: Correlations, path analysis and correlated response to selection. Journal of Agriculture (2): 337 - 342.

Panhwar, S.A., Baloch, M.J., Jatoi, W.A. Veesar, N.F. and Majeedano, M.S. 2008. Combining ability estimates from line $\mathrm{x}$ tester mating design in upland cotton. Proceedings of the Pakistan Academy of Sciences 45(2):69 - 74.

Sharma, H.C., Dhillon, M.K. and Pampapathy, G. 2006. Multiple-resistance to sorghum shoot fly, spotted stem borer and sugarcane aphid in sorghum. International Journal of Tropical Insect Science 26(4):239 - 245.

Songa, J.M., Guofa, Z. and Overholt, W.A. 2001. Relationships of stem borer damage and plant physical condition to maize yield in a semiarid zone of eastern Kenya. Insect Science and Its Application 21(3):243 249.

Wale, M., Schulthess, F., Kairu, E.W. and Omwega, C.O. 2007. Cereal yield losses caused by lepidopterous stem borers at different nitrogen fertilizer rates in Ethiopia. Journal of Appllied Entomology 130(4):220 - 229. 